\title{
Grand Challenges in Wireless Communications
}

\author{
Daniel Benevides da Costa ${ }^{1 *}$ and Hong-Chuan Yang ${ }^{2}$ \\ ${ }^{1}$ Department of Computer Engineering, Federal University of Ceará, Sobral, Brazil, ${ }^{2}$ Department of Electrical and Computer \\ Engineering, University of Victoria, Victoria, BC, Canada
}

Keywords: spectral efficiency, energy efficiency, security, interference management, global connectivity

\section{INTRODUCTION}

By enabling tetherless connectivity first between people, then between people and the Internet, wireless communications fundamentally transformed our daily life and created a fully connected society. Over the past two decade, mass-offering of mobile broadband (MBB) access to the Internet has been the dominating theme of wireless communications and recently became a reality with introduction of various advanced transmission technologies, including multiple antenna transmission and reception (MIMO), multicarrier transmission, channel-adaptive transmission, etc. These technological developments also establish a solid foundation for the future enhancement of MBB services (Zhang et al., 2016).

The growing demand of extremely high-data-rate services, together with emerging advanced Internet of Things (IoT) and Industry 4.0 applications bring new technical challenges to wireless communications. New service classes, such as ultra-HD video and multisensory virtual reality, push for even higher spectral efficiency, and the exploration of extreme frequency bands. While accommodating fast-growing enhanced MBB services, future wireless systems need to simultaneously support the communication demands of massive IoT devices as well as various mission-critical equipment (IMT vision, 2015). Advanced IoT applications require ultra-reliable low-latency and high energy efficient communications for miscellaneous connected devices. Future human-centric services demand accurate localization and multi-dimensional sensing. The full integration of computing, communication, and control with the application artificial intelligence and machine learning is essential to the realization of Industry 4.0 (Wollschlaeger et al., 2017).

Future wireless communication system should effectively support a universal and ubiquitous cyber physical infrastructure for a huge variety of applications with novel network structure, spectrum access schemes, and resource allocation solutions, while taking into account the energy efficiency and security/privacy considerations (Dang et al., 2020). To effectively deliver ultra-high data rate, massive connectivity, and seamless coverage, while accommodating dramatically different quality of service requirements, highly innovative technological solutions are required to address various challenges facing wireless communications. Several grand challenges inherent to future wireless systems are outlined in the following.

\section{GRAND CHALLENGE 1: SECURITY, SECRECY, AND PRIVACY}

Received: 10 June 2020

Accepted: 06 July 2020

Published: 22 July 2020

Wireless communication systems are carrying a growing amount of confidential information. The broadcasting nature of wireless transmissions, however, makes the transmitted information vulnerable to eavesdropping. To effectively support various highly-secrecy-sensitive applications, novel transmission technologies should apply to facilitate or even enhance the information secrecy. For example, the security performance of wireless transmission could be improved by exploring wireless propagation characteristics, as in physical layer security technology (Shiu et al., 2011). 
The challenge is how to maximally benefit from such exploration while respecting the privacy of legitimate users. Innovative transmission solutions to secrecy and privacy enhancement are of great continuing interests to the wireless community (Nour et al., 2020).

\section{GRAND CHALLENGE 2: RESOURCE AND SPECTRUM UTILIZATIONS}

The spectrum suitable for wireless communications is becoming increasingly scarce, which motivate the exploration of new spectrum bands, including millimeter-wave and tera-Hertz (THz). While enjoying larger bandwidth, the coverage area over these higher RF bands are generally much smaller due to their higher propagation loss. The most popular mitigation solution is to apply directional beamforming transmission technology. The challenge is how to efficiently adjust beamforming directions with the changing environment, especially in a multiuser scenario (Zhang et al., 2019). The design of cost-effective THz wireless transceivers is another practical challenge to be addressed. Another solution to address spectrum scarcity is to improve the utilization of existing spectrum through cognitive radio transmission. Secondary access of under-utilized radio spectrum can accommodate IoT applications with less stringent quality of service requirements. Meanwhile, the success deployment of secondary system relies upon efficient spectrum sensing and channel estimation algorithms for resource constrained IoT devices (Zhu et al., 2020).

\section{GRAND CHALLENGE 3: COMMUNICATION INFRASTRUCTURE}

Another direction to accommodate the growing demand of wireless services is to improve system resource utilization with novel system structure. Large-scale collaborative cellfree network structure can overcome the limitations of static resource allocation of traditional cellular systems (Buzzi et al., 2020). Through joint signal processing enabled by cloud computing, multiple base stations will collaboratively serve multiple users using distributed antenna arrays, achieving high spectrum utilization efficiency and dynamically responding to the changes in service demands and propagation environments. Both small cell implementation and relay-assisted systems can be considered as the special cases of the general cellfree concept. The challenge is how to realize the potential performance gains with acceptable system complexity and implementation cost, associated with real-time channel estimation, synchronization in time and frequency domains, and joint processing at fully-connected base stations (Jin et al., 2019). Significant research efforts are being carried out to find the best tradeoff between performance, complexity, and cost. Furthermore, in order to provide heterogeneous global connectivity envisaged to future wireless systems, the integration of satellite, aerial, terrestrial, and underwater networks has become an emerging trend of communication infrastructure and will attract significant attention and studies along the next years. While enabling huge connectivity benefits, this global integration will face unprecedented challenges due to its proper peculiarities, such as heterogeneity, self-organization, and time-variability. In this case, the efficient cross-layer and system integration design, resource management and network optimization, and tractable analytical frameworks for coverage and other performance evaluations are of paramount importance for the successful implementation of these emerging communication infrastructures.

\section{GRAND CHALLENGE 4: ENERGY EFFICIENCY ENHANCEMENT}

Many IoT applications involve a huge number of resourcelimited sensors, which are expected to autonomously operate over 10 years. Although transmitting in a sporadic fashion, these sensor nodes demand transmission schemes with very high energy efficiency. Improving the channel condition during transmission will naturally help reduce transmission energy consumption. Massive MIMO beamforming (Prasad et al., 2017) and large intelligent surface (Wu and Zhang, 2019) are two of the potential technologies to create favorable propagation environment. Meanwhile, obtaining the channel knowledge and optimally configuring the antenna arrays in an efficient fashion are practical challenges faced by these technologies. In certain application scenarios, these sensor nodes may eliminate transmission energy consumption through backscattering transmitted signals from other sources, as used in RFID devices. While some early works have shown promising results (Duan et al., 2017), many challenges need to be tackled before backscatter communications transceiver can be applied in practical applications.

\section{GRAND CHALLENGE 5: INTEGRATION OF WIRELESS INFORMATION AND POWER TRANSFER}

Due to enormous energy consumption expected by the massive number of connected nodes in future wireless networks, several strategies have been proposed to implement self-sustainable communication systems ( $\mathrm{Ku}$ et al., 2016). Specifically, the integration of wireless information and power transfer (WIPT) enables proactive energy replenishment of wireless devices and becomes a promising solution to power energy-constrained wireless networks ( $\mathrm{Lu}$ et al., 2015) which will be particularly important for future wireless networks since next generation of IoT devices will be even more power-hungry due to the huge computation needs for intelligent processing. However, WIPT presents some technical challenges and some few examples are given next. The transmission region of wireless power transfer is too limited. Maximum energy efficiency that can be achieved in the far-field usually do not exceed 50\%. Therefore, further studies are needed in the far-field zone to improve directivity and energy transmission efficiency in different communication technologies. Moreover, harmful interference can be viewed as useful energy source in WIPT context (Zhu et al., 2015). In 
this case, how to mitigate interference and, at the same time, facilitate energy transfer, which may be conflicting, require an in-depth analysis. The effect of mobility is another factor that should be considered in WIPT systems owing to timevarying feature, in which resource allocation needs to be dynamic and adaptive. The impact on health requires also better investigations due to safety concerns on deploying highfrequency transmissions. Particularly, careful investigations are needed to examine the safety of terahertz radiation (Dang et al., 2020). Circuit design is another practical challenge, mainly because of energy harvesting and power transfer components need to be small enough to be embedded in lowpower devices. Then, the integration of wireless information and power transfer still has a long road until its successful practical implementation, and it is envisaged that some of these current concerns can be addressed along the next couple of years.

\section{GRAND CHALLENGE 6: WIRELESS ACCESS TECHNIQUES}

Effective wireless access techniques are essential to future generations of wireless communication systems to fundamentally meet the stringent requirements, which include very high spectral efficiency, very low latency, massive device connectivity, very high achievable data rate, ultrahigh reliability, excellent user fairness, high throughput, support to diverse quality-of-service (QoS), energy efficiency, and a dramatic reduction in the cost (Yang et al., 2019). Non-orthogonal multiple access (NOMA) has been recognized as a strong candidate due to its intrinsic features to fulfill such heterogeneous demands (Ding et al., 2017). By exploiting the non-orthogonality design, NOMA is able to support more users than the number of available time-, frequency-, or code-domain resources, increasing consequently the spectral efficiency and promoting a massive connectivity with a limited number of resource blocks. Moreover, relying on different power allocation strategies, NOMA guarantees user fairness in addition to ensure that all users' quality-ofservices can be met. NOMA has also been applied together with wireless caching (Ding et al., 2018), buffer-aided relaying systems (Zhang et al., 2017), and short-packet transmissions (Sun et al., 2018) to enable ultra-reliable and low-latency communications. Another promising wireless access technique refers to rate-splitting, which appears as another powerful nonorthogonal transmission strategy with a robust interference management (Clerckx et al., 2020). The main idea is to decode part of the interference while treating the remaining portion as noise, which differs from the two conventional interference mitigation schemes that either fully treat interference as noise or fully decode interference. Challenges behind those nonorthogonal strategies concern mainly to the reduction of the implementation complexity (Makki et al., 2020), integration with emerging applications, such as intelligent reflecting surfaces and unmanned aerial vehicles (UAV), and security provisioning, in order to make them appropriate for being applied in practical systems.

\section{GRAND CHALLENGE 7: DYNAMIC ARCHITECTURE AND NETWORK FUNCTIONS ANALYSIS}

Future wireless networks are envisaged to have a dynamic topology in nature because of network densification, in which users will have many connections so that user association decision will become a tricky part. For instance, vehicular systems move quickly and this extreme dynamic environment (e.g., dynamic cell and topology shaping) will change the interference patterns rapidly. Characterizing the environment dynamics in order to ensure that the system takes full advantage of this new topology will be crucial in the next generation of wireless networks (Tarik et al., 2019). Moreover, due to the dynamic behavior of devices joining and leaving wireless systems, maintaining privacy and anonymity will be a challenge for being considered in future wireless networks (Viswanathan and Mogensen, 2020). New mathematical models and tools will also be required to provide an accurate analysis and optimization of systems operating in such dynamic topology. It is also expected that future wireless networks can realize fast and self-intelligent development with a dynamic deployment of the network functions (Chen et al., 2020). Furthermore, new cognitive radio-inspired technologies which enable an intelligent dynamic spectrum access are envisaged. Another point is how to design a network architecture to tackle with the dynamic cooperation among terminals in order to meet users' requirements, to support seamless and ubiquitous communications during the movement, and to enhance spectrum efficiency and user experience. The use of UAVs to support dynamic network topologies and emergency communications will play a key role for the successful implementation of these architectures and network functions. However, to this end, numerous networking challenges need to be properly tackled, such as new protocols accounting for high mobility, dynamic topology, intermittent low-latency links, and power constraints. Last, but not least, dynamic network slicing will be a key management driver when a massive number of users are connected to a high number of heterogenous networks (Chowdury et al., 2019). Such a strategy will allow network operators to use dedicated virtual networks with the aim to support the optimized delivery of services toward to a huge number of users, machines, and vehicles.

\section{GRAND CHALLENGE 8: CODING AND MODULATION}

New coding and modulation schemes are crucial in future wireless networks to ensure that the achievable gains predicted by theory can be realized in practice. Specifically, advanced channel coding and modulation schemes as well as space-airground-underwater integrated networks can help to truly fulfill the massive connectivity (everywhere and every time) (Yuan et al., 2019). Moreover, to provide a much higher spectral efficiency in future wireless networks, a good strategy is to adopt better modulation and channel coding schemes or new modulation resources. Along the technological evolving, each 
generation was dominated by a new coding scheme. For instance, convolutional codes were considered in $2 \mathrm{G}$, turbo codes and enhanced turbo codes were adopted in $3 \mathrm{G}$ and $4 \mathrm{G}$, respectively, and LDPC and polar codes were employed in 5G. Most of these considered codes were designed with limited capabilities in terms of coding rate, which means that the performance of the codes was optimized for specific code rates. In this case, enhancements in the existing coding schemes are expected to improve the performance as well as to make it robust to different environments. Moreover, modulation and channel schemes can be jointly designed in order to minimize the information loss incurred when they are built separately, i.e., when detection and decoding are carried out disconnectedly. In this case, how to design channel coding, modulation, detection and decoding with high data rates, low latency, high reliability, and larger bandwidths deserves an in-depth investigation in order to ensure its successful implementation in the next generation of wireless networks (Latva-aho and Keppännen, 2019).

\section{GRAND CHALLENGE 9: RESOURCE AND INTERFERENCE MANAGEMENT}

In order to support various emerging applications, new resource and interference management schemes are expected in future wireless networks. Under this perspective, machine learningbased techniques have attracted huge interest due to their ability to improve system performance and reduce the computational cost. A real-time management is another feature which makes ML-based strategies a promising tool for attending the stringent demands required to new future applications and services (Tarik

\section{REFERENCES}

Buzzi, S., D’Andrea, C., Zappone, A., and D’Elia, C. (2020). User-centric 5G cellular networks: resource allocation and comparison with the cell-free massive MIMO approach. IEEE Trans. Wireless Commun. 19, 1250-1264. doi: 10.1109/TWC.2019.2952117

Chen, S., Liang, Y.-C., Sun, S., Kang, S., Chen, W., and Peng, M. (2020). Vision, Requirements, and Technology Trends of $6 G-$ How to Tackle Challenges of System Coverage, Capacity, User Data-rate and Movement Speed. Available online at: https://arxiv.org/abs/2002.04929

Chowdury, M. Z., Shahjalal, M., Ahmed, S., and Jang, Y. M. (2019). 6 G Wireless Communication Systems: Applications, Requirements, Technologies, Challenges, and Research Directions. Available online at: https://arxiv.org/abs/1909. 11315

Clerckx, B., Mao, Y., Schober, R., and Poor, H. V. (2020). Rate-splitting unifying SDMA, OMA, NOMA, and multicasting in MISO broadcast channel: a simple two-user rate analysis. IEEE Wireless Commun. Lett. 9, 349-353. doi: 10.1109/LWC.2019.2954518

Dang, S., Amin, O., Shihada, B., and Alouini, M.-S. (2020). What should 6G be? Nat. Electron. 3, 20-29. doi: 10.1038/s41928-019-0355-6

Ding, Z., Fan, P., Karagiannidis, G. K., Schober, R., and Poor, H. V. (2018). NOMA assisted wireless caching: strategies and performance analysis. IEEE Trans. Commun. 66, 4854-4876. doi: 10.1109/TCOMM.2018. 2841929

Ding, Z., Lei, X., Karagiannidis, G. K., Schober, R., Yuan, J., and Bhargava, V. K. (2017). A survey on non-orthogonal multiple access for $5 \mathrm{G}$ networks: research challenges and future trends. IEEE J. Sel. Areas Commun. 35, 2181-2195. doi: 10.1109/JSAC.2017.2725519 et al., 2019). In such a case, the network will be engineered with distributed ML techniques embedded in several terminals, and how quickly they adapt to new conditions of the network will be an important measure. Furthermore, ultra-dense deployment of future wireless networks with small cells will bring big challenges in terms of inter-cell interference and how to efficiently deal with it will require careful investigations (Yastrebova et al., 2018).

\section{CONCLUSIONS}

Many technical challenges are to be addressed to satisfying stringent and conflicting requirements for future wireless systems, including very high spectral efficiency, very low latency, massive device connectivity, very high achievable data rate, ultra-high reliability, excellent user fairness, high throughput, diverse quality-of-service, energy efficiency, and a dramatic cost reduction. The field of wireless communications will witness many exciting technological breakthroughs in the foreseeable future. Cross-discipline discussions and collaborations of academia as well as industries will be key components to drive these breakthroughs. To support such multi-disciplinary activities, the "Wireless Communication" section of Frontier in Communications and Networking plans to serve as an open platform for idea generation, refinements, and perfection and to contribute to the development of a universal and ubiquitous cyber physical infrastructure for a fully-connected world.

\section{AUTHOR CONTRIBUTIONS}

All authors listed have made a substantial, direct and intellectual contribution to the work, and approved it for publication.

Duan, R., Jäntti, R., Yiǧitler, H., and Ruttik, K. (2017). On the achievable rate of bistatic modulated rescatter systems. IEEE Trans. Veh. Technol. 66, 9609-9613. doi: 10.1109/TVT.2017.2715421

IMT vision (2015). Framework and Overall Objectives of the Future Development of IMT for 2020 and Beyond. Technical report, ITU-R Report. M.2083.

Jin, Y., Zhang, J., Jin, S., and Ai, B. (2019). Channel estimation for cell-free mmWave massive MIMO through deep learning. IEEE Trans. Veh. Technol. 68, 10325-10329. doi: 10.1109/TVT.2019.2937543

Ku, M.-L., Li, W., Chen, Y., and Liu, K. J. R. (2016). Advances in energy harvesting communications: past, present, and future challenges. IEEE Commun. Surveys Tut. 18, 1384-1412. doi: 10.1109/COMST.2015.2497324

Latva-aho, M., and and Keppännen, K. (2019). "Key drivers and research challenges for 6G ubiquitous wireless intelligence (White Paper)," in 6G Flagship.

Lu, X., Wang, P., Niyato, D., Kim, D. I., and Han, Z. (2015). Wireless networks with RF energy harvesting: a contemporary survey. IEEE Commun. Surveys Tut. 17, 757-789. doi: 10.1109/COMST.2014.2368999

Makki, B., Chitti, K., Behravan, A., and Alouini, M.-S. (2020). A survey of NOMA: current status and open research challenges. IEEE Open J. Commun. Soc. 1, 179-189. doi: 10.1109/OJCOMS.2020.2969899

Nour, B., Sharif, K., Li, F., and Wang, Y. (2020). Security and privacy challenges in information-centric wireless internet of things networks. IEEE Secur. Privacy 18, 35-45. doi: 10.1109/MSEC.2019.2925337

Prasad, K. N. R. S. V., Hossain, E., and Bhargava, V. K. (2017). Energy efficiency in massive MIMO-based 5G networks: opportunities and challenges. IEEE Wireless Commun. 24, 86-94. doi: 10.1109/MWC.2016.1500374WC

Shiu, Y.-S., Chang, S. Y., Wu, H.-C., Huang, S. C.-H., and Chen, H.H. (2011). Physical layer security in wireless networks: a tutorial. 
IEEE Wireless Commun. 18, 66-74. doi: 10.1109/MWC.2011.575 1298

Sun, X., Yan, S., Yang, N., Ding, Z., Shen, C., and Zhong, Z. (2018). Short-packet downlink transmission with non-orthogonal multiple access. IEEE Trans. Wireless Commun. 7, 4550-4564. doi: 10.1109/TWC.2018.2827368

Tarik, F., Khandaker, M. R. A., Wong, K.-K., Imran, M., Bennis, M., and Debbah, M. (2019). A Speculative Study on 6G. Available online at: https://arxiv.org/abs/1902.06700

Viswanathan, H., and Mogensen, P. (2020). Communications in the 6G era. IEEE Access 8, 57063-57074. doi: 10.1109/ACCESS.2020.2981745

Wollschlaeger, M., Sauter, T., and Jasperneite, J. (2017). The future of industrial communication: automation networks in the era of the internet of things and industry 4.0. IEEE Indus. Electron. Mag. 11, 17-27. doi: 10.1109/MIE.2017.2649104

Wu, Q., and Zhang, R. (2019). Towards smart and reconfigurable environment: intelligent reflecting surface aided wireless network. IEEE Commun. Mag. 58, 106-112. doi: 10.1109/MCOM.001.1900107

Yang, K., Yang, N., Ye, N., Jia, M., Gao, Z., and Fan, R. (2019). Non-orthogonal multiple access: achieving sustainable future radio access. IEEE Commmun. Mag. 57, 116-121. doi: 10.1109/MCOM.2018.1800179

Yastrebova, A., Kirichek, R., Kouncheryavy, Y., Borodin, A., and Kouncheryavy, A. (2018). "Future networks 2030: Architectures and requirements," in 10th Int. Congress Ultra Modern Telecommunication Control System (ICUMT) (Moscow)

Yuan, Y., Zhao, Y., Zhong, B., and Parolari, S. (2019). Potential Key Technologies for $6 G$ Mobile Communications. Available online at: https://arxiv.org/ftp/arxiv/papers/1910/1910.00730.pdf
Zhang, Q., Liang, Z., Li, Q., and Qin, J. (2017). Buffer-aided non-orthogonal multiple access relaying systems in Rayleigh fading channels. IEEE Trans. Commun. 65, 95-106. doi: 10.1109/TCOMM.2016.2630050

Zhang, X., Chen, L., Qiu, J., and Abdoli, J. (2016). On the waveform for 5G. IEEE Commun. Mag. 54, 74-80. doi: 10.1109/MCOM.2016.1600337CM

Zhang, Z., Xiao, Y., Ma, Z., Xiao, M., Ding, Z., Lei, X., et al. (2019). 6G wireless networks: Vision, requirements, architecture, and key technologies. IEEE Veh. Technol. Mag. 14, 28-41. doi: 10.1007/978-3-030-01150-5

Zhu, G., Zhong, C., Suraweera, H. A., Karagiannidis, G. K., Zhang, Z., and Tsiftsis, T. A. (2015). Wireless information and power transfer in relay systems with multiple antennas and interference. IEEE Trans. Commun. 63, 1400-1418. doi: 10.1109/TCOMM.2015.2398862

Zhu, P., Li, J., Wang, D., and You, X. (2020). Machine-learningbased opportunistic spectrum access in cognitive radio networks. IEEE Wireless Commun. 27, 38-44. doi: 10.1109/MWC.001.190 0234

Conflict of Interest: The authors declare that the research was conducted in the absence of any commercial or financial relationships that could be construed as a potential conflict of interest.

Copyright $\odot 2020$ da Costa and Yang. This is an open-access article distributed under the terms of the Creative Commons Attribution License (CC BY). The use, distribution or reproduction in other forums is permitted, provided the original author(s) and the copyright owner(s) are credited and that the original publication in this journal is cited, in accordance with accepted academic practice. No use, distribution or reproduction is permitted which does not comply with these terms. 